\title{
Mass spectrometry-based identification of a B-cell maturation antigen-derived T-cell epitope for antigen-specific immunotherapy of multiple myeloma
}

\author{
Tatjana Bilich $0^{1,2}$, Annika Nelde ${ }^{1,2}$, Jens Bauer ${ }^{1,2}$, Simon Walz ${ }^{2,3}$, Malte Roerden ${ }^{4}$, Helmut R. Salih ${ }^{1}$, Katja Weisel ${ }^{4,5}$, \\ Britta Besemer ${ }^{4}$, Ana Marcu², Maren Lübke ${ }^{2}$, Juliane Schuhmacher ${ }^{2}$, Marian C. Neidert ${ }^{6}$, Hans-Georg Rammensee ${ }^{2,7}$, \\ Stefan Stevanović, ${ }^{2,7}$ and Juliane S. Walz ${ }^{1}$
}

\begin{abstract}
The B-cell maturation antigen (BCMA) is currently being evaluated as promising tumor-associated surface antigen for T-cell-based immunotherapy approaches, such as CAR T cells and bispecific antibodies, in multiple myeloma (MM). Cytotoxic T cells bearing BCMA-specific T-cell receptors might further allow targeting HLA-presented antigens derived from the intracellular domain of BCMA. By analyzing a mass spectrometry-acquired immunopeptidome dataset of primary MM samples and MM cell lines for BCMA-derived HLA ligands, we identified the naturally presented HLA-B*18restricted ligand $P(B C M A)_{B^{*} 18}$. Additionally, $P(B C M A)_{B^{*} 18}$ was identified on primary $C L L$ samples, thereby expanding the range for possible applications. $\mathrm{P}(\mathrm{BCMA})_{\mathrm{B}^{*} 18}$ induced multifunctional $\mathrm{BCMA}$-specific cells de novo from naïve $\mathrm{CD} 8^{+}$ $T$ cells of healthy volunteers. These T cells exhibited antigen-specific lysis of autologous peptide-loaded cells. Even in the immunosuppressive context of $M M$, we detected spontaneous memory T-cell responses against $P(B C M A)_{B^{*} 18}$ in patients. By applying CTLA-4 and PD-1 inhibition in vitro we induced multifunctional $P(B C M A)_{B^{*} 1} 8^{-s p e c i f i c ~} C D 8^{+} T$ cells in MM patients lacking preexisting BCMA-directed immune responses. Finally, we could show antigen-specific lysis of autologous peptide-loaded target cells and even MM.1S cells naturally presenting $\mathrm{P}(\mathrm{BCMA})_{\mathrm{B}^{*} 18}$ using patient-derived

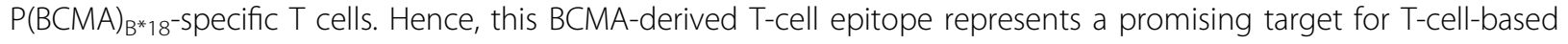
immunotherapy and monitoring following immunotherapy in B-cell malignancy patients.
\end{abstract}

\section{Introduction}

T-cell-based immunotherapies for multiple myeloma (MM) are on the advance, further improving the outcome of this still incurable disease. Beside strategies like allogeneic stem cell transplantation ${ }^{1}$, donor lymphocyte infusion $^{2}$, immunomodulatory drugs ${ }^{3}$, and immune checkpoint inhibitors ${ }^{4}$, that induce a fairly general $\mathrm{T}$-cell

\footnotetext{
Correspondence: Juliane S. Walz (juliane.walz@med.uni-tuebingen.de) 'University Hospital Tübingen, Clinical Collaboration Unit Translational Immunology, German Cancer Consortium (DKTK), Tübingen, Germany

University of Tübingen, Institute for Cell Biology, Department of Immunology, Tübingen, Germany
}

Full list of author information is available at the end of the article activation, several advanced approaches for targeting myeloma cells more specifically are in development. Such antigen-specific immunotherapy approaches comprise mono- and bispecific antibodies ${ }^{5,6}$, chimeric antigen receptor (CAR) $\mathrm{T}$ cells ${ }^{7}, \mathrm{~T}$-cell receptor (TCR)-engineered $\mathrm{T}$ cells ${ }^{8}$, and dendritic cell (DC)- or peptide-based vaccines $^{9,10}$. A main prerequisite for such approaches is the selection of feasible targets for MM-directed T-cell responses. Ideally, such targets should be presented both, exclusively and frequently on myeloma cells. A particularly promising target for antigen-specific immunotherapy in $\mathrm{MM}$ and other B-cell malignancies is the B-cell maturation antigen (BCMA, TNFRSF17, CD269), a 
member of the tumor necrosis receptor superfamily. BCMA is a preferentially B-lineage-restricted differentiation transmembrane protein with exclusive presentation on myeloma cells, plasma blasts, differentiated plasma cells, and late memory B cells ${ }^{6}$.

Currently, several therapeutic approaches targeting BCMA are being evaluated in preclinical settings and clinical trials including CAR $\mathrm{T}$ cells ${ }^{7}$, bispecific antibodies and other T-cell engagers ${ }^{6,11}$, antibody-drug conjugates ${ }^{12}$, or immunotoxins ${ }^{13}$. Particularly BCMA-targeting CAR T cells showed promising results in heavily pretreated and refractory MM patients with an overall response rate of $81 \%{ }^{7}$. However, despite these encouraging results, potential toxicities and immense costs should be taken into account, in particular prior to application of such immunotherapy approaches in elderly and comorbid MM patients or larger patient cohorts at an earlier disease setting ${ }^{14,15}$. Furthermore, all these substances are targeting the extracellular domain of BCMA with an associated risk of surface antigen $\operatorname{loss}^{16}$. Cytotoxic $\mathrm{T}$ cells bearing BCMA-specific TCRs might be an alternative approach to target MM cells. Such TCRs can also recognize intracellular proteins or domains, which are processed and presented via human leukocyte antigen (HLA) molecules on the surface of tumor cells ${ }^{17}$. Antigen-specific $\mathrm{T}$ cells can either be induced in vivo by low side effect vaccination-based approaches or generated ex vivo as TCR-engineered cells. The main prerequisite for these approaches is the identification and characterization of naturally presented HLA-restricted peptides, which can serve as target structures for $\mathrm{T}$ cells ${ }^{18}$. In a previous study, we characterized the naturally presented immunopeptidome of MM using a mass spectrometry (MS)-based approach and identified several novel MM-associated antigens ${ }^{19}$. Here, we evaluated this dataset for the presence of BCMA-derived peptides to provide a proof of concept for the feasibility to identify and target naturally presented $\mathrm{T}$ cell epitopes from intracellular domains of highly promising tumor surface antigens.

\section{Results}

\section{MS-based identification of BCMA-derived HLA-presented peptides in MM}

Previously acquired MS datasets ${ }^{19,20}$ of primary MM samples and MM cell lines (MCLs) were reprocessed using the search engine SequestHT and evaluated for the presence of naturally presented BCMA-derived peptides. Analysis of the immunopeptidome of seven primary MM samples and five MCLs revealed a total of 17633 unique HLA class I ligands from 7627 different source proteins as well as 9482 unique HLA class II peptides from 2371 source proteins. We identified two BCMA-derived HLA class I-restricted ligands, both derived from its intracellular domain (Fig. 1a). The HLA-B*18-restricted peptide DEIILPRGL, referred to as $\mathrm{P}(\mathrm{BCMA})_{\mathrm{B}^{*} 18}$, was identified in $17 \%$ (2/12 samples, one primary MM patient sample and the MCL MM.1S) of the analyzed MM immunopeptidomes with a remarkably high allotypeadjusted frequency of $67 \%$ (2/3 HLA-B* $18^{+}$samples). Notably, $\mathrm{P}(\mathrm{BCMA})_{\mathrm{B}^{*} 18}$ showed $\mathrm{MM}$ - and B-lineageassociated presentation and was solely detected on $1 / 5$ benign B-cell (20\%) and 2/17 benign lymph node samples (12\%) according to our extensive benign immunopeptidome database (149 297 HLA class I ligands; 17093 source proteins; 404 samples from various tissues). Additionally, $\mathrm{P}(\mathrm{BCMA})_{\mathrm{B}^{*} 18}$ could also be identified in the immunopeptidome of $2 / 3$ (67\%) primary HLA-B*18 chronic lymphocytic leukemia (CLL) samples ${ }^{21}$. In contrast, the HLA-B*40-restricted $\mathrm{P}(\mathrm{BCMA})_{\mathrm{B}^{*} 40}$ ligand TEIEKSISA was detected solely in 1/12 (8\%) MM-derived samples with an allotype-adjusted frequency of $33 \%(1 / 3$ HLA-B $* 40^{+}$samples) but displayed no selective MMassociation due to its representation in a variety of benign tissues. Furthermore, we identified two HLA class IIrestricted BCMA-derived antigens that showed MMexclusive presentation according to our benign HLA class II immunopeptidome database (214 908 HLA class II peptides; 15840 source proteins; 366 samples from various tissues). However, these HLA class II-restricted BCMA-derived peptides were both detected only in MCLs but not in primary MM samples with a low representation frequency of $8 \%$ (1/12 samples) in our MM cohort.

Therefore, we selected the $\mathrm{P}(\mathrm{BCMA})_{\mathrm{B}^{*} 18}$ peptide due to its $\mathrm{MM}$-association and the high representation frequency for further immunological characterization. Prior to immunogenicity testing, we validated the experimentally acquired spectrum of $\mathrm{P}(\mathrm{BCMA})_{\mathrm{B}^{*} 18}$ by comparison of MS/MS spectra as well as of the reversed-phase retention times of the precursor ions using an isotope-labeled synthetic peptide (Fig. 1b).

\section{$\mathrm{P}(\mathrm{BCMA})_{\mathrm{B}^{*} 18}$ induced multifunctional peptide-specific $T$ cells in healthy volunteers in vitro}

To assess the immunogenicity of $\mathrm{P}(\mathrm{BCMA})_{\mathrm{B}^{*} 18}$, we performed in vitro artificial antigen-presenting cell (aAPC)based priming experiments using $\mathrm{CD}^{+} \mathrm{T}$ cells of healthy volunteers (HVs). Effective de novo priming and expansion of antigen-specific $\mathrm{T}$ cells was observed in $100 \%$ of analyzed HVs $(n=10)$ with frequencies of peptide-specific T cells ranging from $0.1-7.9 \%$ (mean $0.9 \%$ ) within the viable $\mathrm{CD} 8^{+}$ T-cell population (Fig. 2a, Supplemental Table 1). The de novo induction of peptide-specific cells was further proven by the lack of preexisting memory $\mathrm{T}$-cell responses after 12-day recall stimulation (Fig. 2a). Priming experiments with a control peptide frequently presented on HLA-B*18 in both, tumor and benign tissues (peptide presentation $>90 \%$ in HLA-matched sources), confirmed MM-specificity of the induced T-cell responses (Fig. 2b, Supplemental Table 2). Furthermore, multifunctionality of the induced 
a

\begin{tabular}{ccccccc}
\hline $\begin{array}{c}\text { Peptide } \\
\text { ID }\end{array}$ & $\begin{array}{c}\text { Peptide } \\
\text { sequence }\end{array}$ & $\begin{array}{c}\text { HLA } \\
\text { restriction }\end{array}$ & $\begin{array}{c}\text { Frequency } \\
\text { in MM samples }\end{array}$ & $\begin{array}{c}\text { Allotype-adjusted frequency } \\
\text { in MM samples }\end{array}$ & $\begin{array}{c}\text { Allotype-adjusted frequency } \\
\text { in CLL samples }\end{array}$ & $\begin{array}{c}\text { Identification } \\
\text { in benign samples }\end{array}$ \\
\hline$P(B C M A)_{B^{*} 18}$ & DEIILPRGL & $\mathrm{B}^{*} 18$ & $2 / 12(17 \%)$ & $2 / 3(67 \%)$ & $2 / 3(67 \%)$ & B cell-containing tissues only \\
\hline$P(B C M A)_{B^{*} 40}$ & TEIEKSISA & $\mathrm{B}^{*} 40$ & $1 / 12(8 \%)$ & $1 / 3(33 \%)$ & $0 / 3(0 \%)$ & several benign samples/tissues \\
\hline P1(BCMA $)_{\text {class II }}$ & DEIILPRGLEY & class II & $1 / 12(8 \%)$ & n.a. & n.a. & no \\
\hline$P 2(B C M A)_{\text {class II }}$ & LEKSRTGDEIILPRG & class II & $1 / 12(8 \%)$ & n.a. & n.a. & no \\
\hline
\end{tabular}

b

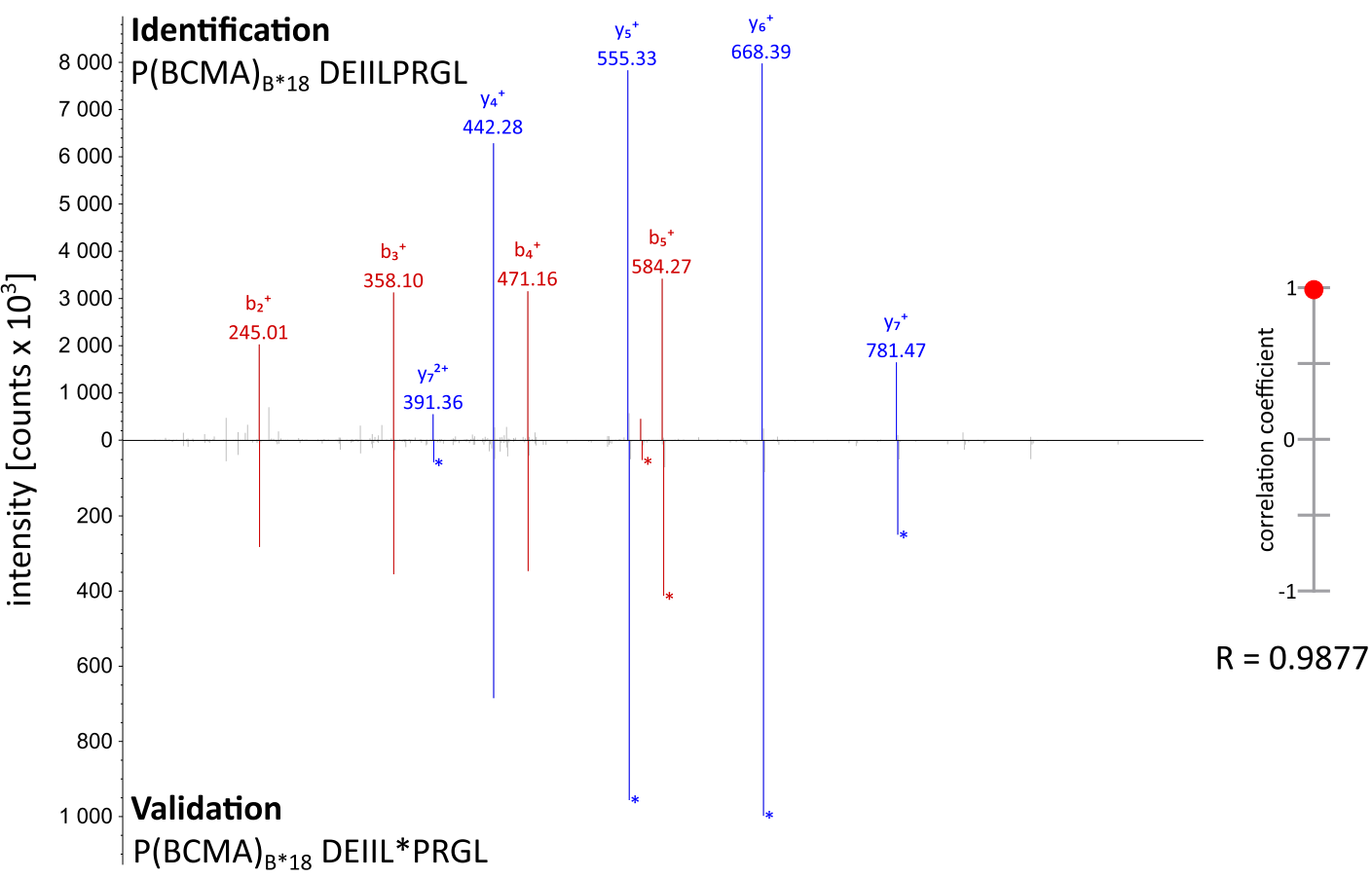

Fig. 1 Identification of $B C M A$-derived peptides and validation of $P(B C M A)_{B^{*} 18}$ using a synthetic isotope-labeled peptide. a Identified BCMA-derived HLA-presented peptides with their respective sequence, HLA restriction, their total and allotype-adjusted frequency in the immunopeptidomes of the MM and CLL cohort, as well as their occurrence in the HLA peptidome of benign tissues. $\mathbf{b}$ Validation of the experimentally eluted $\mathrm{P}(\mathrm{BCMA})_{\mathrm{B}^{*} 18}$ peptide using the corresponding synthetic isotope-labeled peptide. Comparison of the fragment spectrum $(\mathrm{m} / \mathrm{z}$ on the $x$-axis) of the $\mathrm{P}(\mathrm{BCMA})_{\mathrm{B}^{*} 18}$ peptide eluted from a primary $\mathrm{MM}$ patient sample (identification) with its corresponding synthetic peptide (validation). The spectrum of the synthetic peptide is mirrored on the $x$-axis. Identified $b$ - and $y$-ions are marked in red and blue, respectively. lons containing the isotope-labeled amino acid are marked with asterisks. The calculated spectral correlation coefficient is depicted on the right graph. ID identification, MM multiple myeloma, CLL chronic lymphocytic leukemia, n.a. not available.

$\mathrm{P}(\mathrm{BCMA})_{\mathrm{B}^{\prime \prime} 18}$-specific $\mathrm{T}$ cells could be demonstrated in $6 / 6$ HV samples. Using intracellular cytokine staining (ICS), we observed a significant production of IFNY and TNF as well as upregulation of the degranulation marker CD107a upon $\mathrm{P}(\mathrm{BCMA})_{\mathrm{B}^{*} 18}$ stimulation (Fig. 2c). Moreover, cytotoxicity assays with polyclonal $\mathrm{P}(\mathrm{BCMA})_{\mathrm{B}^{*} 18 \text {-specific effector } \mathrm{T} \text { cells }}$ revealed their capacity to induce antigen-specific lysis of autologous $\mathrm{CD} 8^{-}$peptide-loaded target cells (Fig. 2d-f).

\section{Detection of preexisting $\mathrm{P}(\mathrm{BCMA})_{\mathrm{B}^{*} 18^{-}}$-specific memory T-cell responses in $\mathrm{MM}$ patients}

We further evaluated the existence of spontaneous preexisting memory $\mathrm{T}$-cell responses directed against $\mathrm{P}(\mathrm{BCMA})_{\mathrm{B}^{\prime \prime 18}}$ in IFN $\gamma$ enzyme-linked immunospot (ELISPOT) assays using peripheral blood mononuclear cells
(PBMCs) of HLA-B*18 $8^{+}$MM patients (Supplemental Table 3). We observed $\mathrm{P}(\mathrm{BCMA})_{\mathrm{B}^{\prime \prime} 18}$-induced IFNY secretion in 1/4 (25\%) samples of MM patients (Fig. 3a). Notably, we could not detect any preexisting immune responses against $\mathrm{P}(\mathrm{BCMA})_{\mathrm{B}^{*} 18}$ in samples from $\mathrm{HVs}$ $(n=12$, Fig. $3 \mathrm{~b})$, further confirming the MM-specificity of $\mathrm{P}(\mathrm{BCMA})_{\mathrm{B}^{\prime \prime} 18}$-directed immune responses.

\section{Combination of immune checkpoint inhibitors and $\mathrm{P}(\mathrm{BCMA})_{\mathrm{B}^{*} 18}$ using cells of $\mathrm{MM}$ patients induced peptide-specific $T$ cells with anti-myeloma activity}

Next, we aimed to overcome the reported profound immune defects (including impaired function of immune effector cells) in $\mathrm{MM}^{22}$, and therefore evaluate the potential of $\mathrm{P}(\mathrm{BCMA})_{\mathrm{B}^{*} 18}$ to induce de novo functional $\mathrm{T}$-cell 


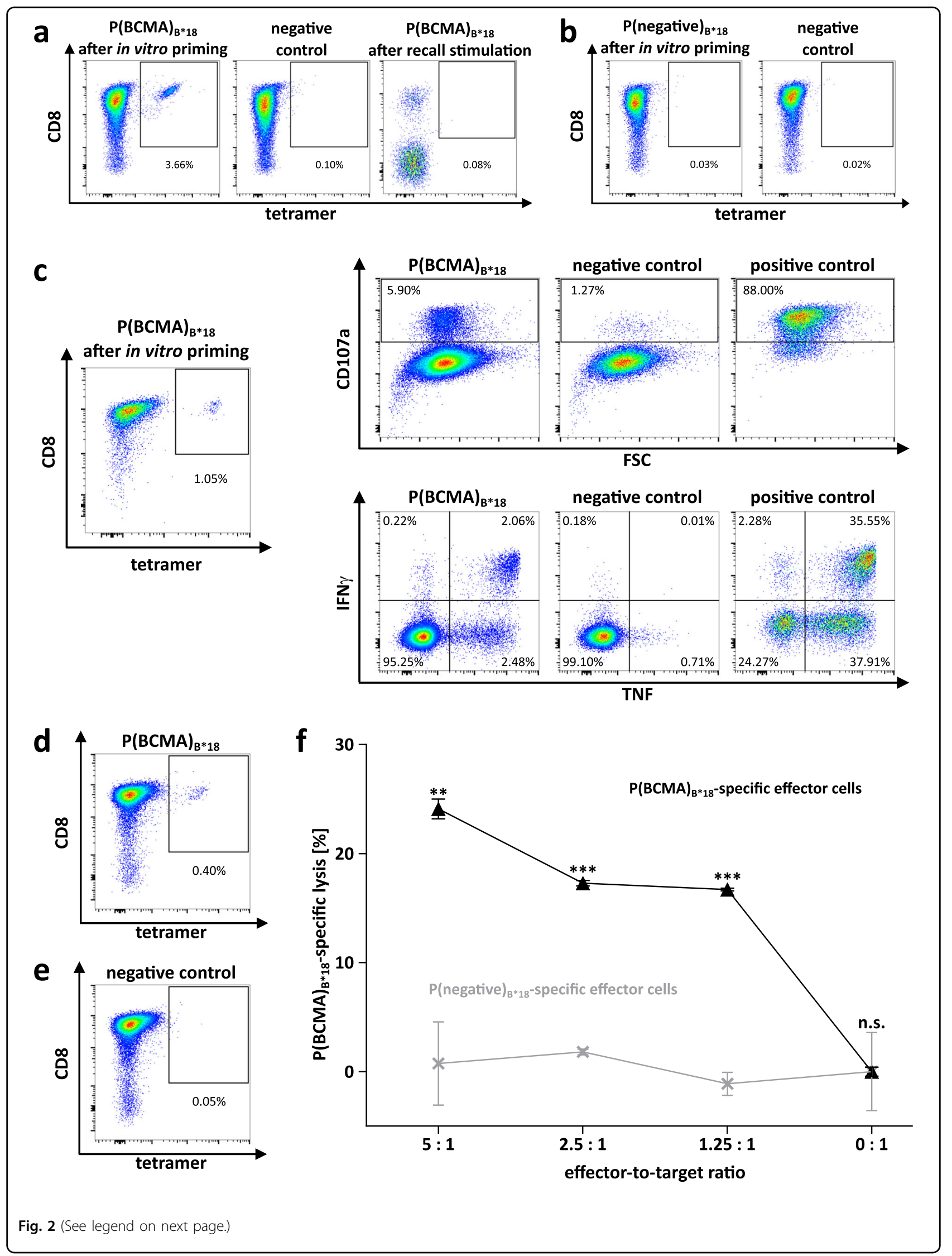


(see figure on previous page)

Fig. 2 Induction and functional characterization of $\mathbf{P}(\mathbf{B C M A})_{\mathbf{B}^{*} 18^{-} \text {-specific } C D 8^{+}} \mathbf{T}$ cells from $\mathrm{HVs}$. a Naïve $\mathrm{CD} 8^{+} \mathrm{T}$ cells from $\mathrm{HVs}$ were primed in vitro using aAPCs. Graphs show single, viable cells stained for CD8 and PE-conjugated multimers of indicated specificity. Tetramer staining was performed

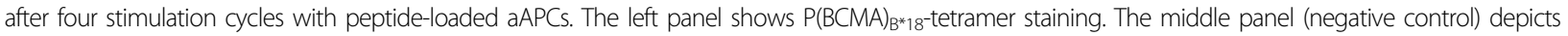

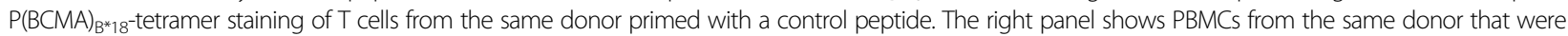
tested negative for preexisting memory T cells following 12-day recall stimulation. $\mathbf{b}$ Tetramer staining after four stimulation cycles with negative control

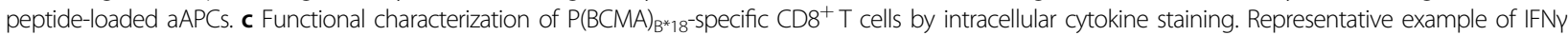

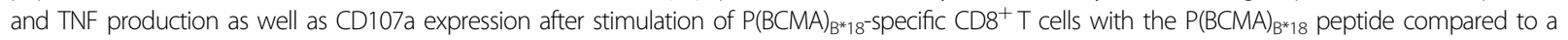

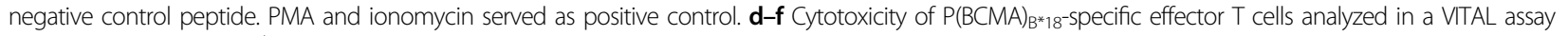
with in vitro primed $C D 8^{+} T$ cells. $\mathbf{d}$, e Tetramer staining of polyclonal effector cells prior to the VITAL assay determined the amount of $P(B C M A)_{B}{ }^{*} 8^{-S p e c i f i c ~}$ effector cells in the $\mathbf{d}$ population of successfully $\mathrm{P}(\mathrm{BCMA})_{\mathrm{B}^{*} 18}$-primed $\mathrm{CD} 8^{+} \mathrm{T}$ cells and in the $\mathbf{e}$ population of control cells from the same donor primed with

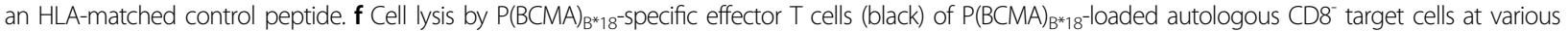

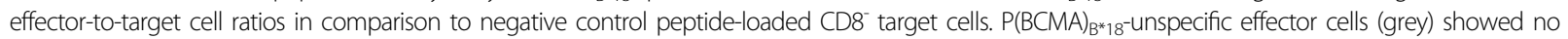
peptide-specific lysis of the same targets. Results are shown as mean \pm SEM for three independent technical replicates. Significance was determined using two-tailed paired student's t-test. FSC forward scatter, n.s. not significant, ${ }^{* *} p<0.01,{ }^{* * *} p<0.001$.

responses using $\mathrm{T}$ cells of $\mathrm{MM}$ patients that displayed no preexisting memory immune responses against $\mathrm{P}(\mathrm{BCMA})_{\mathrm{B}^{* 18}}$ following 12-day recall stimulation. While no

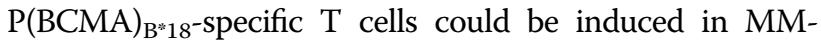
derived samples using our standard priming protocol, we observed de novo induction of $\mathrm{P}(\mathrm{BCMA})_{\mathrm{B}^{* 1} 18^{-}}$-specific $\mathrm{T}$ cells with frequencies of $0.2-4.1 \%$ (mean 1.6\%) within the $\mathrm{CD} 8^{+}$ T-cell population of an MM patient upon addition of CTLA-4 and PD-1 blocking antibodies (Fig. 4a). These

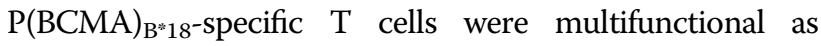
demonstrated by IFNY and TNF production as well as upregulation of CD107a (Fig. 4b) and peptide-specific lysis of autologous $\mathrm{P}(\mathrm{BCMA})_{\mathrm{B}^{*} 18^{-}}$-loaded $\mathrm{CD} 8^{-}$cells (Fig. 4c-e). Moreover, MM-derived $\mathrm{P}(\mathrm{BCMA})_{\mathrm{B}^{*} 18}$-specific $\mathrm{CD} 8^{+}$ $\mathrm{T}$ cells showed specific lysis of MM.1S cells (Fig. $4 \mathrm{f}-\mathrm{h}$ ), which naturally present $\mathrm{P}(\mathrm{BCMA})_{\mathrm{B}^{*} 18}$ as detected by MSbased immunopeptidomics.

Taken together, we identified a naturally presented myeloma-associated, BCMA-derived peptide, which constitutes a promising and highly immunogenic target for tailored T-cellbased immunotherapy and monitoring of immunotherapeutic approaches in MM and other B-cell malignancies.

\section{Discussion}

Valid antigen targets are the main prerequisite for the development of clinically effective antigen-specific cancer immunotherapy. These highly promising target structures are represented by HLA-independent surface antigens, such as BCMA in MM. However, the number of such antigens is limited due to the required exclusive presentation on the surface of tumor cells ${ }^{23}$. HLA-dependent antigens, in contrast, can originate from any intracellular protein or domain and are not restricted to cell surface proteins. Therefore, the amount of potential HLA-dependent targets for a given tumor entity is expected to be considerably higher. In recent years, numerous studies have defined HLA ligands derived from tumor-specific mutations as main targets of immune checkpoint inhibitor-induced T-cell responses in solid tumors $^{24,25}$. Nonetheless, only a very small proportion of
DNA-level mutations results in naturally presented, mutation-derived neoantigens detectable in the HLA ligandome ${ }^{26,27}$. Hence, the role of neoantigens for the development of broadly applicable immunotherapy, especially in low mutational burden cancer entities such as MM, remains unclear. We and others have previously described several non-mutated HLA ligands as pathophysiologically relevant targets for T-cell-based immunotherapy approa$\mathrm{ches}^{28}$, especially for tumors with a low mutational burden, such as hematological malignancies ${ }^{19,21,29-31}$.

In this study we aimed to identify HLA-dependent antigen targets derived from the intracellular domain of the established surface antigen BCMA using an MS-based approach. We identified $\mathrm{P}(\mathrm{BCMA})_{\mathrm{B}^{*} 18}$ as a highly immunogenic naturally presented epitope capable of inducing potent and multifunctional cytotoxic $\mathrm{T}$-cell responses. This is in line with recent data reporting on the high immunogenicity of computationally predicted HLA 02 -restricted T-cell epitopes derived from the extracellular surface domain of $\mathrm{BCMA}^{32,33}$. Our mass spectrometric approach further allowed us to validate $\mathrm{P}(\mathrm{BCMA})_{\mathrm{B}^{*} 18}$ additionally as a target for CLL, for which plasma BCMA-levels were described as a prognostic factor $^{34}$. However, so far less data are available demonstrating the utility of BCMA-based immunotherapeutic approaches in CLL. As already shown on gene and protein expression level presentation of BCMA-derived peptides is not restricted to myeloma and CLL cells but is-albeit less frequently-detectable in benign B-cell containing tissues $^{35}$. This is in line with other B-lineage-specific targets such as CD19, for which antigen-specific therapies, including CAR $\mathrm{T}$ cells and bispecific antibodies, are already approved for clinical use ${ }^{36,37}$. As the expression of CD19 is not limited to malignant B cells, targeting of CD19 can lead to on-target/off-tumor side effects including for example hypogammaglobulinemia, which however are manageable in the clinical setting ${ }^{38}$.

Our approach for the identification of BCMA-derived $\mathrm{T}$-cell epitopes might be translated to other MM-associated 

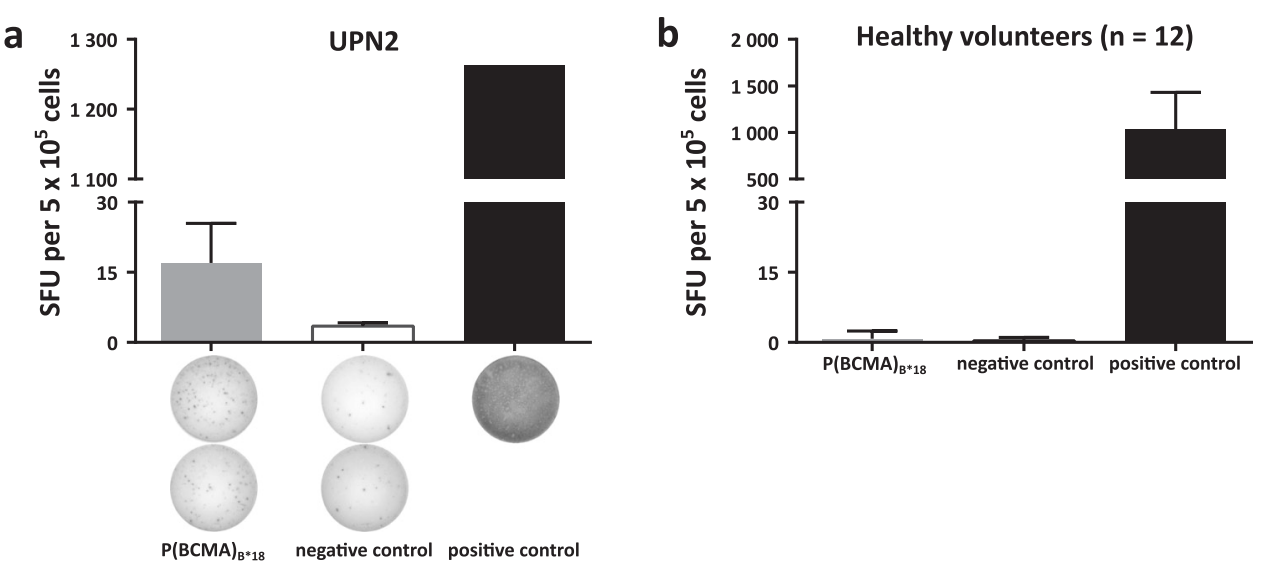

Fig. 3 Preexisting memory T-cell responses directed against BCMA detected in IFN $\gamma$ ELISPOT assays. a, b Memory T-cell responses directed against $\mathrm{P}(\mathrm{BCMA})_{\mathrm{B}^{*} 18}$ were evaluated in IFNY ELISPOT assays after 12-day recall stimulation using PBMCs of a MM patients or $\mathbf{b} \mathrm{HVs}$. PHA was used as positive control. The peptide DEVRTLTY served as negative control. Data are expressed as mean \pm SD of two independent technical replicates for the MM patient and as mean \pm SD of all $12 \mathrm{HVs}$ analyzed in two independent technical replicates each. UPN uniform patient number, HVs healthy volunteers, SFU spot forming unit.

antigens such as SLAMF7, CD38, CD74, or CD138 ${ }^{39}$, providing naturally processed HLA-presented ligands derived from intracellular domains of established membrane-bound tumor-associated antigens. Such HLA ligands represent promising targets for (i) low side effect single agent vaccine approaches in elderly patients or early disease states ${ }^{21,28,40}$, (ii) TCR-engineered $\mathrm{T}$ cells ${ }^{41}$, (iii) antibody-based approaches ${ }^{42}$, or (iv) novel approaches combining HLA-dependent and -independent antigens and treatments ${ }^{43}$. In addition, such $\mathrm{T}$-cell epitopes can be used for the assessment and monitoring of $\mathrm{T}$-cell responses following various types of antigen-specific, or as in this example BCMA-specific, immunotherapy approaches. However, the HLA allotype restriction of HLA-peptide targets, in terms of HLA-B*18 covering only about $12 \%$ of the world population ${ }^{44}$, represents a limitation concerning the development of broadly applicable immunotherapy approaches. This calls for combinatorial approaches using peptides of various HLA restrictions or for patient (group)-individualized approaches. However, in this context it has to be taken into account, that different HLA ligands with distinct HLA allotype restrictions derived from the same source protein can show different tissue distribution as demonstrated exemplarily for the $\mathrm{P}(\mathrm{BCMA})_{\mathrm{B}^{*} 40}$ peptide in this study. The lack of correlation between protein expression and the immunopeptidome can be explained by the differential protein processing in malignant cells along with the complexity of HLA ligand formation, which is frequently altered in tumor cells ${ }^{45,46}$.

Besides the selection of optimal target antigens for the development of clinically effective T-cell-based immunotherapies in $\mathrm{MM}$, one must consider the profound immune defects that prevail in these patients and might impair the efficacy of inducing antigen-specific T-cell responses in vivo ${ }^{22,47}$. A major immunosuppressive mechanism in $\mathrm{MM}$ is the upregulation of inhibitory immune checkpoint molecules on $\mathrm{T}$ cells ${ }^{22,39}$. By employing PD-1/CTLA-4 blockade, we were able to overcome this mechanism and to induce multifunctional cytotoxic $\mathrm{P}(\mathrm{BCMA})_{\mathrm{B} " 18}$-specific T cells, even in MM-derived samples. These data are in line with reports from other solid tumors and hematological malignancies ${ }^{48-50}$, pointing towards a combination of antigen-specific immunotherapy with general approaches optimizing immune responses, as exemplified with immune checkpoint blockade ${ }^{51,52}$.

Taken together, this study provides first evidence that intracellular domain-derived HLA ligands from MMassociated membrane antigens can provide a novel category of highly immunogenic antigen targets for tailored combinatorial immunotherapies for patients suffering from MM or other B-cell malignancies.

\section{Methods}

Patients, blood samples, and cell lines

PBMCs collected from MM patients at the Department of Hematology and Oncology (Tübingen, Germany), and from healthy volunteers, were isolated by density gradient centrifugation using Biocoll (Biochrom GmbH, Berlin, Germany). Informed consent was obtained from all subjects in accordance with the Declaration of Helsinki protocol. The study was performed according to the guidelines of the local ethics committee. Patient characteristics are provided in Supplemental Table 3. The MM.1S (ATCC CRL-2974) and MV4-11 (DSMZ ACC102) cell lines were tested for mycoplasma contamination and cultured in the recommended cell media as described previously ${ }^{20}$. 


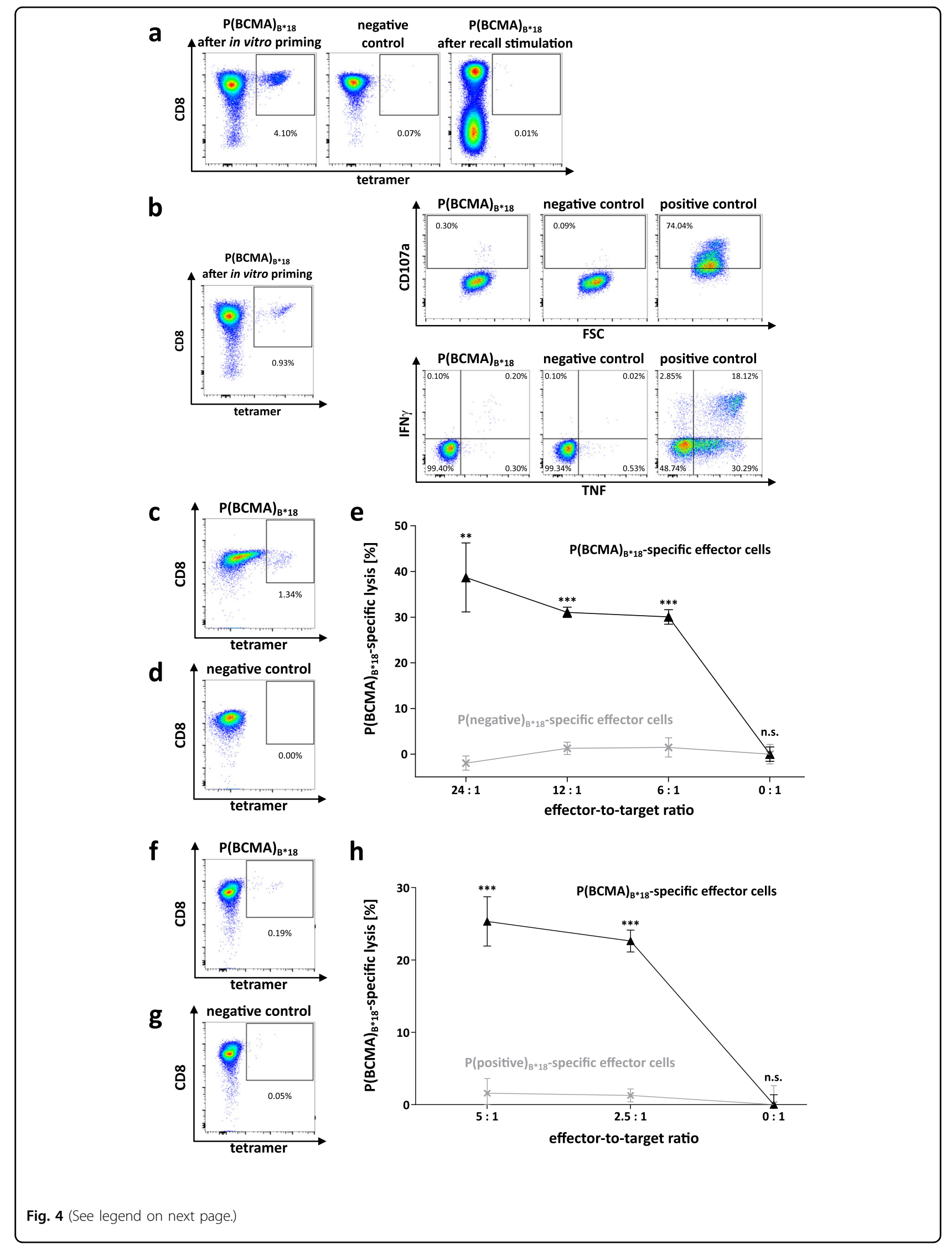


(see figure on previous page)

Fig. 4 Induction and functional characterization of $P(B C M A)_{B^{*} 18^{-s p e c i f i c ~} C D 8^{+}} \mathbf{T}$ cells from a myeloma patient. a Naïve $C D 8^{+} T$ cells from UPN1 were stimulated four times with peptide-loaded aAPCs in addition to PD-1 and CTLA-4 blocking antibodies. Graphs show single, viable cells stained for $C D 8$ and PE-conjugated multimers of indicated specificity. The left panel shows $P(B C M A)_{B^{*} 18}$-tetramer staining. The middle panel

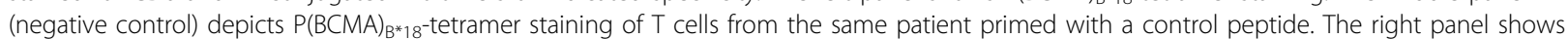
PBMCs from the same patient that were tested negative for preexisting memory T cells following 12-day recall stimulation. $\mathbf{b}$ Example of IFNY and TNF production as well as CD107a expression following stimulation with the $\mathrm{P}(\mathrm{BCMA})_{\mathrm{B}^{*} 18}$ peptide compared to the negative control peptide using peptide-specific cells of UPN1. PMA and ionomycin served as positive control. $\mathbf{c}, \mathbf{d}, \mathbf{f}, \mathbf{g}$ Tetramer staining of polyclonal effector cells prior to the VITAL

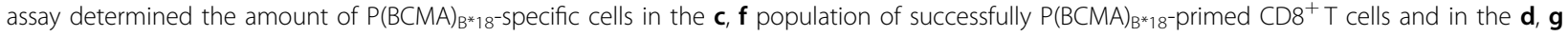

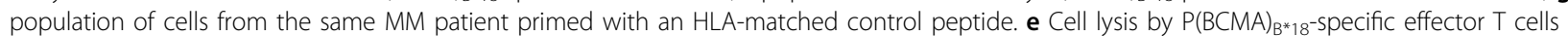
(black) of $\mathrm{P}(\mathrm{BCMA})_{\mathrm{B}^{*} 18^{-}}$-loaded autologous $\mathrm{CD}^{-}$target cells at various effector-to-target cell ratios in comparison to $\mathrm{P}$ (negative) B $^{*} 18$ control peptide-

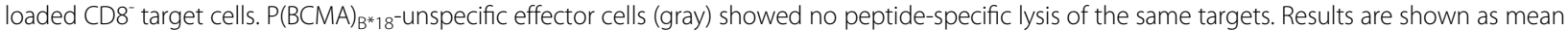

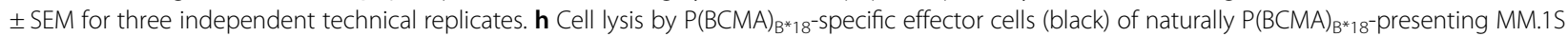
target cell line at various effector-to-target cell ratios in comparison to MV4-11 P(BCMA $)_{\mathrm{B}^{*} 18}$-negative target cell line. $\mathrm{P}(\mathrm{BCMA})_{\mathrm{B}^{*} 18^{8} \text {-unspecific effector }}$ $T$ cells (gray) showed no peptide-specific lysis of the same targets. Results are shown as mean \pm SEM for three independent technical replicates. Significance was determined using two-tailed paired student's $t$-test. FSC forward scatter, UPN uniform patient number, n.s. not significant, ${ }^{* *} p<0.01$, ${ }^{* * *} p<0.001$.

\section{MS data reevaluation}

Reprocessing and reanalyzing of previously acquired and published MS data of primary MM patient samples and MCLs $^{19,20}$ was performed as described before ${ }^{53}$. The Proteome Discoverer (v1.3, Thermo Fisher Scientific, Waltham, MA, USA $)^{54}$ was used to integrate the search results of the SequestHT search engine (University of Washington, Seattle, WA, USA) against the human proteome (Swiss-Prot database ${ }^{55}$ ) without enzymatic restriction. Precursor mass tolerance was set to $5 \mathrm{ppm}$ and fragment mass tolerance to $0.5 \mathrm{Da}$. Oxidized methionine was allowed as dynamic modification. The false discovery rate, estimated by the Percolator algorithm $2.04^{56}$, was limited to $5 \%$ for HLA class I and 1\% for HLA class II. Peptide lengths were set to 8-12 or 8-25 amino acids for HLA class I and II, respectively. HLA class I annotation was performed using SYFPEITHI $1.0^{57}$ and NetMHCpan $4.0^{58}$.

\section{Spectrum validation}

Spectrum validation of the experimentally eluted peptide was performed by computing the similarity of the spectrum with the corresponding synthetic isotope-labeled peptide measured in a complex matrix. The spectral correlation coefficient was calculated between the spectra of the eluted and the synthetic peptide considering the mass shift of ions containing the isotope-labeled amino acid $^{59}$. Peptides were synthesized as described previously ${ }^{30}$.

\section{aAPC priming of naïve $\mathrm{CD}^{+} \mathrm{T}$ cells}

Priming of cytotoxic $\mathrm{T}$ lymphocytes was conducted using aAPCs following magnetic-activated cell-sorting (MACS) for $\mathrm{CD}^{+}$cells as described previously ${ }^{30}$. In detail, 800000 streptavidin-coated microspheres were loaded with $200 \mathrm{ng}$ biotinylated HLA:peptide monomer and $600 \mathrm{ng}$ biotinylated anti-human CD28 monoclonal antibody (mAb, clone 9.3, in-house production). These aAPCs were used for the stimulation of $1 \times 10^{6} \mathrm{CD}^{+}$
T cells in four stimulation cycles in addition to $5 \mathrm{ng} / \mathrm{mL}$ of IL-12 (PromoKine, Heidelberg, Germany). For MM patient-derived $\mathrm{CD}^{+}$cells the stimulation protocol comprised addition of $1 \mu \mathrm{g} / \mathrm{mL}$ purified anti-human PD-1 (CD279, \#14-2799-80) and CTLA-4 (CD152, \#16-152982, Invitrogen, Carlsbad, CA, USA) mAbs directly after PBMC isolation, after MACS, as well as for the first and third aAPC stimulation.

\section{Cytokine and tetramer staining}

Frequencies of peptide-specific $\mathrm{CD}^{+} \mathrm{T}$ cells were determined by tetramer staining using the $\mathrm{PE} / \mathrm{Cy} 7$ antihuman CD8 mAb (\#737661, Beckman Coulter, Brea, CA, USA) and $5 \mu \mathrm{g} / \mathrm{mL}$ of PE-labeled HLA:peptide tetramer (in-house production). Tetramers of the same HLA allotype containing irrelevant control peptides were used as negative control (Supplemental Table 2).

Functionality of peptide-specific $T$ cells was analyzed by ICS using PE/Cy7 anti-human CD8, PacificBlue antihuman TNF (\#502920), FITC anti-human CD107a (\#328606, BioLegend, San Diego, CA, USA), and PE antihuman IFNY (\#506507, BD, Franklin Lakes, NJ, USA) antibodies as described previously ${ }^{30}$.

Results of tetramer staining were considered positive if the frequency of peptide-specific $\mathrm{CD}^{+} \mathrm{T}$ cells was $\geq 0.1 \%$ of viable cells and at least three-fold higher than the frequency of peptide-specific cells in the negative control according to the harmonization guidelines for HLApeptide multimer assays of the international Cancer Vaccine Consortium proficiency panel ${ }^{60}$. The same evaluation criteria were applied for ICS. All samples were analyzed using a FACS-Canto ${ }^{\mathrm{TM}}$ II cytometer and FlowJo software version 10.0.7 (BD).

\section{Cytotoxicity assays}

Peptide-specific $\mathrm{CD}^{+} \mathrm{T}$ cells were analyzed for their capacity to induce peptide-specific target cell lysis in the 
flow cytometry-based VITAL assay ${ }^{30}$. Autologous CD8 target cells were either loaded with the $\mathrm{P}(\mathrm{BCMA})_{\mathrm{B}^{\prime \prime} 18}$ peptide or an irrelevant control peptide (Supplemental Table 2) and labeled with CFSE or FarRed (life technologies, Carlsbad, CA, USA), respectively. The $\mathrm{P}(\mathrm{BCMA})_{\mathrm{B}^{*} 18^{-} \text {-specific }}$ effector cells were added in the indicated effector-to-target ratios. Specific lysis of peptide-loaded CD8 target cells was calculated relative to control targets. Additionally, we performed the VITAL assay using the $\mathrm{P}(\mathrm{BCMA})_{\mathrm{B}^{*} 18}$-presenting MM.1 S cell line stained with CFSE as target cells. The HLA-B* $18^{+}$acute myeloid leukemia cell line MV4-11, negative for $\mathrm{P}(\mathrm{BCMA})_{\mathrm{B}^{*} 18}$ in mass spectrometry-based HLA ligandome analysis, was stained with FarRed and served as negative target cell line.

\section{Amplification of peptide-specific T cells and IFN $\gamma$ ELISPOT assay}

PBMCs of MM patients and HVs were pulsed with $1 \mu \mathrm{g} / \mathrm{mL}$ per peptide and cultured for 12-days. In addition, cells were supplemented on day 3, 5, and 7 with 20 U/mL IL-2 (Novartis, Basel, Switzerland). On day 12, PBMCs were analyzed in the IFN $\gamma$ ELISPOT assay as described previously ${ }^{30}$ using an ImmunoSpot S5 analyzer (CTL Europe $\mathrm{GmbH}$, Bonn, Germany). The negative control peptide $\mathrm{P}$ (negative) $)_{\mathrm{B}^{\prime \prime} 18}$ (Supplemental Table 2) was used to exclude unspecific immune responses, for example from NK cells. Preexisting memory T-cell responses were considered positive if $\geq 10$ spots/well were counted and the mean spot count was at least three-fold higher than the mean spot count of the negative control.

\section{Acknowledgements \\ We thank Ulrike Schmidt, Cécile Gouttefangeas, Claudia Falkenburger, Patricia Hrstić, Nicole Bauer, Beate Pömmerl, and Ulrich Wulle for excellent technical support. This work was supported by the German Cancer Consortium (DKTK); the Deutsche Forschungsgemeinschaft (DFG, German Research Foundation) under Grant STI 704/1-1; the Deutsche Forschungsgemeinschaft under Germany's Excellence Strategy under Grant EXC2180-390900677; the Wilhelm Sander Stiftung under Grant 2016.177.1; the José Carreras Leukämie-Stiftung under Grant DJCLS 05 R/2017; the Bundesministerium für Bildung und Forschung (BMBF) under Grant 031A535A; the European Union (EU) under Grant ERC AdG339842 MUTAEDITING.}

\section{Author details}

${ }^{1}$ University Hospital Tübingen, Clinical Collaboration Unit Translational Immunology, German Cancer Consortium (DKTK), Tübingen, Germany. ${ }^{2}$ University of Tübingen, Institute for Cell Biology, Department of Immunology, Tübingen, Germany. ${ }^{3}$ University Hospital Tübingen, Department of Urology, Tübingen, Germany. ${ }^{4}$ University Hospital Tübingen, Department of Hematology and Oncology, Tübingen, Germany. ${ }^{5}$ University Hospital Hamburg-Eppendorf, Department of Oncology, Hamburg-Eppendorf, Germany. 'University Hospital Zurich and University of Zurich, Department of Neurosurgery, Clinical Neuroscience Center, Zurich, Switzerland. ${ }^{7}$ German Cancer Consortium (DKTK), DKFZ partner site Tübingen, Tübingen, Germany

\section{Author contributions}

T.B., A.N., H.-G.R., S.S. and J.S.W. designed the study; T.B., A.N., J.B. and A.M. performed reanalysis of the previously acquired MS dataset; T.B., S.W., M.L. and J.S. conducted in vitro T-cell experiments; S.W., M.R., H.R.S., K.W., B.B., M.C.N. and J.S.W. provided new reagents, analytic tools, and samples; S.W., M.R., H.R.S., K.W.,
B.B., M.C.N. and J.S.W. conducted patient data collection and medical evaluation; T.B., A.N., J.B., S.S. and J.S.W. analyzed data; T.B., A.N., J.B. and J.S.W. drafted the manuscript; H.-G.R., S.S. and J.S.W. supervised the study.

\section{Ethics approval and consent to participate}

Informed consent was obtained in accordance with the Declaration of Helsinki protocol. The study was performed according to the guidelines of the local ethics committee (454/2016BO2).

\section{Conflict of interest}

Hans-Georg Rammensee is shareholder of Immatics Biotechnologies GmbH and Curevac AG. The other authors declare no competing financial interests.

\section{Publisher's note}

Springer Nature remains neutral with regard to jurisdictional claims in published maps and institutional affiliations.

Supplementary Information accompanies this paper at (https://doi.org/ 10.1038/s41408-020-0288-3).

Received: 25 August 2019 Accepted: 28 October 2019

Published online: 28 February 2020

\section{References}

1. Krishnan, A. et al. Autologous haemopoietic stem-cell transplantation followed by allogeneic or autologous haemopoietic stem-cell transplantation in patients with multiple myeloma (BMT CTN 0102): a phase 3 biological assignment trial. Lancet Oncol. 12, 1195-1203 (2011).

2. Lokhorst, H. M. et al. The occurrence of graft-versus-host disease is the major predictive factor for response to donor lymphocyte infusions in multiple myeloma. Blood 103, 4362-4364 (2004).

3. Dimopoulos, M. et al. Lenalidomide plus dexamethasone for relapsed or refractory multiple myeloma. N. Engl. J. Med. 357, 2123-2132 (2007).

4. Bezman, N. A. et al. PD-1 blockade enhances elotuzumab efficacy in mouse tumor models. Blood Adv. 1, 753-765 (2017).

5. Lonial, S. et al. Elotuzumab therapy for relapsed or refractory multiple myeloma. N. Engl. J. Med. 373, 621-631 (2015).

6. Seckinger, A. et al. Target expression, generation, preclinical activity, and pharmacokinetics of the BCMA-T cell bispecific antibody EM801 for multiple myeloma treatment. Cancer Cell 31, 396-410 (2017).

7. Brudno, J. N. et al. T cells genetically modified to express an anti-B-cell maturation antigen chimeric antigen receptor cause remissions of poor-prognosis relapsed multiple myeloma. J. Clin. Oncol. 36, 2267-2280 (2018).

8. Mastaglio, S. et al. NY-ESO-1 TCR single edited stem and central memory $\mathrm{T}$ cells to treat multiple myeloma without graft-versus-host disease. Blood $\mathbf{1 3 0}$ 606-618 (2017).

9. Rosenblatt, J. et al. Vaccination with dendritic cell/tumor fusion cells results in cellular and humoral antitumor immune responses in patients with multiple myeloma. Blood 117, 393-402 (2011)

10. Nooka, A. K. et al. Assessment of safety and immunogenicity of PVX-410 vaccine with or without lenalidomide in patients with smoldering multiple myeloma: a nonrandomized clinical trial. JAMA Oncol. 4, e183267 (2018).

11. Hipp, S. et al. A novel BCMAVCD3 bispecific T-cell engager for the treatment of multiple myeloma induces selective lysis in vitro and in vivo. Leukemia $\mathbf{3 1}$ 1743-1751 (2017).

12. Trudel, S. et al. Targeting B-cell maturation antigen with GSK2857916 antibody-drug conjugate in relapsed or refractory multiple myeloma (BMA117159): a dose escalation and expansion phase 1 trial. Lancet Oncol. 19, 1641-1653 (2018)

13. Shancer, Z. et al. Anti-BCMA immunotoxins produce durable complete remissions in two mouse myeloma models. Proc. Natl Acad. Sci. USA 116, 4592-4598 (2019)

14. Titov, A. et al. The biological basis and clinical symptoms of CAR-T therapyassociated toxicites. Cell Death Dis. 9, 897 (2018).

15. Hay, A. E. \& Cheung, M. C. CAR T-cells: costs, comparisons and commentary. J. Med. Econ. 1, 613-615 (2019). 
16. Orlando, E. J. et al. Genetic mechanisms of target antigen loss in CAR19 therapy of acute lymphoblastic leukemia. Nat. Med. 24, 1504-1506 (2018).

17. Falk, K., Rotzschke, O., Stevanovic, S., Jung, G. \& Rammensee, H. G. Allelespecific motifs revealed by sequencing of self-peptides eluted from MHC molecules. Nature 351, 290-296 (1991).

18. Rammensee, H. G. \& Singh-Jasuja, H. HLA ligandome tumor antigen discovery for personalized vaccine approach. Expert. Rev. Vaccines 12, 1211-1217 (2013)

19. Walz, S. et al. The antigenic landscape of multiple myeloma: mass spectrometry (re)defines targets for T-cell-based immunotherapy. Blood 126 1203-1213 (2015).

20. Kowalewski, D. J. et al. Carfilzomib alters the HLA-presented peptidome of myeloma cells and impairs presentation of peptides with aromatic C-termini. Blood. Cancer J. 6, e411 (2016).

21. Kowalewski, D. J. et al. HLA ligandome analysis identifies the underlying specificities of spontaneous antileukemia immune responses in chronic lymphocytic leukemia (CLL). Proc. Natl Acad. Sci. USA 112, E166-E175 (2015).

22. Costa, F., Das, R., Kini Bailur, J., Dhodapkar, K. \& Dhodapkar, M. V. Checkpoint inhibition in myeloma: opportunities and challenges. Front. Immunol. 9, 2204 (2018).

23. Bauer, J., Nelde, A., Bilich, T. \& Walz, J. S. Antigen targets for the development of immunotherapies in leukemia. Int. J. Mol. Sci. 20, 1397 (2019).

24. van Rooij, N. et al. Tumor exome analysis reveals neoantigen-specific T-cell reactivity in an ipilimumab-responsive melanoma. J. Clin. Oncol. 31, e439-e442 (2013).

25. Snyder, A. et al. Genetic basis for clinical response to CTLA-4 blockade in melanoma. N. Engl. J. Med. 371, 2189-2199 (2014).

26. Finn, O. J. \& Rammensee, H. G. Is it possible to develop cancer vaccines to neoantigens, what are the major challenges, and how can these be overcome? neoantigens: nothing new in spite of the name. Cold Spring Harb. Perspect. Biol. 10, a028829 (2018).

27. Ott, P. A. et al. An immunogenic personal neoantigen vaccine for patients with melanoma. Nature 547, 217-221 (2017).

28. Hilf, N. et al. Actively personalized vaccination trial for newly diagnosed glioblastoma. Nature 565, 240-245 (2019).

29. Berlin, C. et al. Mapping the HLA ligandome landscape of acute myeloid leukemia: a targeted approach toward peptide-based immunotherapy. Leukemia 29, 647-659 (2015).

30. Bilich, T. et al. The HLA ligandome landscape of chronic myeloid leukemia delineates novel T-cell epitopes for immunotherapy. Blood 133, 550-65. (2019).

31. Schuster, $\mathrm{H}$. et al. The immunopeptidomic landscape of ovarian carcinomas. Proc. Natl Acad. Sci. USA 114, E9942-E9951 (2017).

32. Bae, J., Samur, M., Richardson, P., Munshi, N. C. \& Anderson, K. C. Selective targeting of multiple myeloma by $B$ cell maturation antigen (BCMA)-specific central memory CD8(+) cytotoxic T Iymphocytes: immunotherapeutic application in vaccination and adoptive immunotherapy. Leukemia 33, 2208-2226 (2019).

33. Bae, J. et al. BCMA peptide-engineered nanoparticles enhance induction and function of antigen-specific CD8(+) cytotoxic T lymphocytes against multiple myeloma: clinical applications. Leukemia 34, 210-223 (2020).

34. Udd, K. A. et al. Plasma B-cell maturation antigen levels are elevated and correlate with disease activity in patients with chronic lymphocytic leukemia. Target Oncol. 14, 551-561 (2019).

35. Carpenter, R. O. et al. B-cell maturation antigen is a promising target for adoptive T-cell therapy of multiple myeloma. Clin. Cancer Res. 19, 2048-2060 (2013).

36. Topp, M. S. et al. Safety and activity of blinatumomab for adult patients with relapsed or refractory B-precursor acute lymphoblastic leukaemia: a multicentre, single-arm, phase 2 study. Lancet Oncol. 16, 57-66 (2015).

37. Turtle, C. J. et al. CD19 CAR-T cells of defined CD4+:CD8+ composition in adult B cell ALL patients. J. Clin. Invest. 126, 2123-2138 (2016).
38. Zugmaier, G. et al. Long-term follow-up of serum immunoglobulin levels in blinatumomab-treated patients with minimal residual disease-positive B-precursor acute lymphoblastic leukemia. Blood Cancer J. 4, 244 (2014).

39. Al-Hujaily, E. M., Oldham, R. A., Hari, P., Medin, J. A. Development of novel immunotherapies for multiple myeloma. Int. J. Mol. Sci. 17, 1506 (2016).

40. Maslak, P. G. et al. Phase 2 trial of a multivalent WT1 peptide vaccine (galinpepimut-S) in acute myeloid leukemia. Blood Adv. 2, 224-234 (2018).

41. Rapoport, A. P. et al. NY-ESO-1-specific TCR-engineered T cells mediate sustained antigen-specific antitumor effects in myeloma. Nat. Med. 21, 914-921 (2015).

42. Dao, T. et al. Therapeutic bispecific T-cell engager antibody targeting the intracellular oncoprotein WT1. Nat. Biotechnol. 33, 1079-1086 (2015).

43. Wu, M. et al. CD19 chimeric antigen receptor-redirected T cells combined with epidermal growth factor receptor pathway substrate 8 peptide-derived dendritic cell vaccine in leukemia. Cytotherapy 21, 659-670 (2019).

44. Bui, H. H. et al. Predicting population coverage of T-cell epitope-based diagnostics and vaccines. BMC Bioinforma. 7, 153 (2006).

45. Hoyos, L. E. \& Abdel-Wahab, O. Cancer-specific splicing changes and the potential for splicing-derived neoantigens. Cancer Cell 34, 181-183 (2018).

46. Mani, A. \& Gelmann, E. P. The ubiquitin-proteasome pathway and its role in cancer. J. Clin. Oncol. 23, 4776-4789 (2005).

47. Kawano, Y., Roccaro, A. M., Ghobrial, I. M. \& Azzi, J. Multiple myeloma and the immune microenvironment. Curr. Cancer Drug Targets 17, 806-818 (2017).

48. Fu, J. et al. Preclinical evidence that PD1 blockade cooperates with cancer vaccine TEGVAX to elicit regression of established tumors. Cancer Res. 74, 4042-4052 (2014)

49. Soares, K. C. et al. PD-1/PD-L1 blockade together with vaccine therapy facilitates effector T-cell infiltration into pancreatic tumors. J. Immunother. 38, 1-11 (2015).

50. Morillon, Y. M. II, Hammond, S. A., Durham, N. M., Schlom, J. \& Greiner, J. W. Enhanced immunotherapy by combining a vaccine with a novel murine GITR ligand fusion protein. Oncotarget 8, 73469-73482 (2017).

51. Massarelli, E. et al. Combining immune checkpoint blockade and tumorspecific vaccine for patients with incurable human papillomavirus 16-related cancer: a phase 2 clinical trial. JAMA Oncol. 5, 67-73 (2019).

52. Ott, P. A. et al. Abstract CT125: A personal neoantigen vaccine, NEO-PV-01, with anti-PD1 induces broad de novo anti-tumor immunity in patients with metastatic melanoma, NSCLC, and bladder cancer. AACR, CT125-CT125 (2018) https://cancerres.aacrjournals.org/content/78/13_Supplement/CT125.

53. Nelde, A. et al. HLA ligandome analysis of primary chronic lymphocytic leukemia (CLL) cells under lenalidomide treatment confirms the suitability of lenalidomide for combination with T-cell-based immunotherapy. Oncoimmunology 7, e1316438 (2018).

54. Eng, J. K. McCormack, A. L. \& Yates, J. R. An approach to correlate tandem mass spectral data of peptides with amino acid sequences in a protein database. J. Am. Soc. Mass Spectrom. 5, 976-989 (1994).

55. UniProt, C. UniProt: a worldwide hub of protein knowledge. Nucleic Acids Res. 47(D1), D506-D515 (2019).

56. Kall, L., Canterbury, J. D., Weston, J., Noble, W. S. \& MacCoss, M. J. Semisupervised learning for peptide identification from shotgun proteomics datasets. Nat. Methods 4, 923-925 (2007).

57. Schuler, M. M., Nastke, M. D. \& Stevanovikc, S. SYFPEITHI: database for searching and T-cell epitope prediction. Methods Mol Biol. 409, 75-93 (2007)

58. Jurtz, V. et al. NetMHCpan-4.0: improved peptide-MHC class I interaction predictions integrating eluted ligand and peptide binding affinity data. $J$. Immunol. 199, 3360-3368 (2017).

59. Toprak, U. H. et al. Conserved peptide fragmentation as a benchmarking tool for mass spectrometers and a discriminating feature for targeted proteomics. Mol. Cell Proteom. 13, 2056-2071 (2014).

60. Britten, C. M. et al. Harmonization guidelines for HLA-peptide multimer assays derived from results of a large scale international proficiency panel of the Cancer Vaccine Consortium. Cancer Immunol. Immunother. 58, 1701-1713 (2009). 